\title{
Poisons, sorciers et contre-sorcellerie en pays Bobo (Haute-Volta)
}

Poisons, witches and antiwitchcraft in the land of the Bobo (Upper Volta)

\section{Guy Le Moal}

\section{(2) OpenEdition}

\section{Journals}

Édition électronique

URL : http://journals.openedition.org/span/172

DOI : $10.4000 /$ span. 172

ISSN : 2268-1558

Éditeur

École pratique des hautes études. Sciences humaines

\section{Édition imprimée}

Date de publication : 1 septembre 1975

Pagination : 78-94

ISSN : 0294-7080

\section{Référence électronique}

Guy Le Moal, «Poisons, sorciers et contre-sorcellerie en pays Bobo (Haute-Volta) », Systèmes de pensée en Afrique noire [En ligne], 1 | 1975, mis en ligne le 04 juillet 2013, consulté le 10 décembre 2020. URL : http://journals.openedition.org/span/172 ; DOI : https://doi.org/10.4000/span.172 


\section{POISONS, SORCIERS ET CONTRE-SORCELLERIE}

en PAYS BOBO. (Haute Volta)

par GuY LE MOAL

PROPOS - On parle peu des sorciers chez les Bobo, ils $n$ 'apparaissent pas au premier plan des préoccupations et il ne semble pas qu'il s'agisse la d'une simple attitude : avec beaucoup d'assurance, on pretend en effet que les perils de la sorcellerie s'ils ont été réels dans le passé, sont jugulés aujourd'hui grâce à l'efficacité des moyens qui ont été mis en oeuvre. Il reste que la contre-sorcellerie, toute triomphante qu'elle soit, est maintenue en activité...

On essaiera ict de préciser ce qu'est le personnaoge du sorcier chez les Bobo et, après quelques reflexions sur le sens de cette contre-sorcellerie qui survit a ses incessantes victoires, on decrira les moyens employés pour détecter et détruire les sorciers.

Chez les Bobo, les sorciers tuent leurs victimes en les empoisonnant. Il s'agit de cette catégorie de sorciers que l'on désigne par le terme anquais de sorcerer. La contre-sorcellerie est extrêmement agissante, elle s'appuie aujourd'hui sur des cultes répandus dans tout le pays. Autrefois, on detectait les sorciers par un "interrogatoire du cadavre". Par la suite, se sont développés les cultes d'esprits particulters, duba et surtout vyetôgo, oui dénoncent les sorciers par le procédé de la possession et provoquent leur mort.

Les Bobo sont maitres en l'art des poisons. Chaque clan et souvent même chacun des liọnages composant ce clan, conserve comme son bien personnel une recette originale qui comprend aussi bien la formule d'un poison que celle de son antidote. Ces poisons-servent a endulre flèches et lances, 11 s sont essentiellement employes dans la chasse et dans la querre. Mais, la science acquise par les Bobo en matiêre de substances vénéneuses ne s'est pas limitée à ces usag̣es en quelque sorte licites, elle a êté détournée, très tôt sans doute, vers des fins 
purement criminelles.

Le poison est chez les Bobo le moyen le plus frequemment utilisé pour attenter a la vie d'autrui. L'homme qui est décide a tuer elabore en secret un poison mortel, très different des poisons violents qu'emploie son lignage pour la chasse et qui provoquent une paralysie foudroyante. Le criminel doit en effet disposer d'un poison à effets lents, capable d'alterer insidieusement la sante de sa victime - ce qui permet de différer le moment de la mort et d'éarer les soupçons.

Sans doute, dans bon nombre de cas, le crime restera occasionnel : une fois sa vengeance accomplie et sa haine tarie, le coupable ne récidive pas. Dans l'esprit des Bobo cependant, il est fermement établi qu'existe une catégorie d'individus infiniment plus redoutables, qui emploient le poison de façon répétée, poussés par une sorte de passion pour le mal. On prête à ces individus certains pouvoirs surnaturels : ils pratiqueraient le culte d'esprits vindicatifs, manieraient des forces malefiques et emprunteraient leurs techniques à la magie - usant de charmes et jetant des sorts - ; mais ce qui reste, de l'avis de tous, constant et caractéristique,c'est l'emplol bien réel qu'ils font du poison pour tuer leurs victimes. Ce sont par-dessus tout des "maîtres du polson" et c'est par ces termes exactement qu'on les désiọne en bobo: kyente (de kyen "poison" et te "propriétaire, maftre de") en dialecte syêkôma des régions du centre ou kyonosố en dialecte syakôma de la région de Bobo Dioulasso.

Peut-on parler de sorcellerie a propos des pratiques du kyente bobo ? Il faut rappeler, au préalable, qu'en français nous ne disposons que du seul terme de "sorcellerie" pour désigner un phénomène exceptionnellement complexe et qui recouvre des types d'agissements et de croyances fort differents, voire même opposés. Les Anglo-saxons, orâce à deux termes, sorcery et witchcraft, opèrent, quant a eux, une distinction aul s'est révêlée pertinente dans de nombreuses ethnies (1) et qui senble

(1) Cf. EVANS-PRITCHARD, E.E. Sorcellerie, oracles et magie chez les $A z$ andé, Paris, Gallimard, 1972. Cf. Éalement:MIDDLETON, J.; WINTER, E.H. éds. Witchcraft and Sorcery in East Africa. London, Rout ledge and Kegan, P., 1963. 
bien rendre compte auss 1 des faits particuliers aux Bobo. Le sorcerer (quilreste par certains côtés encore proche du magicien) n'est pas le dépositaire d'un don herréditaire, il aquit volontairement et avec une pleine conscience de ce qu'il fait, suivant en cela un but personnel et conforme à son intérêt, mais surtout il opère à l'aide d'une technique qui fait appel à des moyens matériels. Le witch au contraire possède un pouvoir inne, 11 agit involontairement et reste inconscient de son etat, falsant le mal pour le mal sans en tirer de bénéfice personnel. Le witch, enfin, n'opère que sur un plan psychique et souvent a distance avec des moyens spirituels d'ordre surnaturel.sorcery et witchcraft (que l'on a rassemblees sous le terme de wizardry. Cf. Middleton, op. cit.) s'opposent à maqie, notamment en ce qu'elles sont toutes deux uniquement orientées vers le mal.

Le witch, être nocturne coúreur de sabbats, monstre psychique capable de se dedoubler et d'agir non pas d'allleurs sur les corps directement, mais sur les principes spirituels qui composent la personne, mangeur d'âmes ou vampire, ce sorcier au sens le plus fort du mot, rien n'indique qu'il ait une existence dans la pensee bobo. Celui qu'on redoute exclusivement c'est "l'empoisonneur", le kyente, et son portrait correspond bien a la description du sorcerer. Le kyente - un homme très généralement - n'a rien dans son apparence physique qui attire l'attention. Si, comme nous l'avons dit, on le soupçonne d'employer quelques moyens magiques et de vouer un culte à des puissances maléfiques, c'est toujours à un moyen matériel, au poison, qu'il fait appel pour donner la mort. Anime par une froide volonté de détruire, c'est en pleine lucidité que le kyente agit, préparant lonquement la mixture mortelle qu'il administrera avec une sournoise habilete, épiant le lent travail du poison et l'irréversible cheminement du mal.

Les Bobo disent generalement que le kyente choisit ses victimes dans le but de satisfaire un ressentiment, ou pour remplir quelque dessein personnel inavouable, mais. on pretend aussi qu'il cède bientôt à la sombre delectation de tuer et n'agit plus dès lors que gratuitement. 
On sait, après les nombreuses etudes qui lui ont été consacrés, que le phénomène de la sorcellerie (aussi bien sorcery que witchcraft) est revélateur de certaines réalités psychologiques et soctales generalement dissimulées; on sait que la sorcellerie est liee à des situations de conflit etqu'elle reflète les contradictions du système social. Aussi les conduites du sorcier, réelles ou imaginaires, apparaissent-elles corme chargées de sens et propres à fournir d'utiles enseignements sur les problèmes spécifiques des individus ou du groupe concerne.

Chez les Bobo, le kyente semble bien n'avoir jamais exercé ses méfaits de façon exclusive à l'intérieur de son lignage, bien plutôt c'est au niveau de la communauté villageoise multi-lignagère qu'il aurait le plus souvent opéré. Cela prend tout son sens si l'on tient, compte du fait que la société bobo se caractérise par la volonté de réaliser des communautés villageoises aussi fortement intégrées que possible, ce qui suppose l'êdification d'équilibres délicats fondés sur des echanges et sur des dosages entre droits et devoirs, entrainant de subtiles tractations et ouvrant le champ à des menées souterraines et à des formes de pression qui sont dans la liqne directe de la sorcellerie. Egalement significatif sans doute est le fait que cette même société bobo n'a pas incarne le mal sous la forme du witch, si riche en fantasmes, mais au'elle a produit le sorcier-empoisonneur calculateur, froid et lucide.

Tout porte à croire que les actes du kyente n'étaient pas, ainsi que feignent de le croire les Bobo, parfaitement gratuits ou même seulement limités a la satisfaction de basses vengeances privees. La sorcel lerie est un instrument d'action - plus souvent d'ailleurs au service du pouvoir et, paradoxalement, des règles établies qu'à celui de la contestation (1).

Parlant du kyente bobo, nous devrions donc être en mesure de dire quelie est sa fonction sociale, quels sont le sens, le but et le résultat de ses actions. Cela ne nous est pourtant

(1) Cf. AUGE, M. "Les croyances à la sorcellerie" in AUGE, M, éd. La construction du Monde, Paris, François Maspero,1974, pp. 52-74. 
guère possible en raison d'une difficulté majeure : il n'y a plus de sorciers en pays bobo!

On affirme en effet de toutes parts qu'après avoir eté pourchassés activement, par les moyens que nous decrirons, les sorciers sont désormais pratiquement éliminés. On remarquera en passant qu'une telle conviction, lorsqu'elle se propage, peut bien se révéler la plus efficace de toutes les armes dans un domaine ou tout procede de l'imaginaire : la croyance en la sorcellerie perd toute assise, dẻs lors qu'on n'adhère plus aux fantasmes dont elle est la somme.

Les choses pourtant ne sont pas si simples. La contresorcellerie est triomphante... mais on constate que partout elle est maintenue en activité, ce qui laisse penser qu'elle a encore quelques raisons d'être. Sans doute apparait-il que la contre-sorcellerie, de curative qu'elle fut par la mise a mort effective d'innombrables sorciers, est devenue préventive : elle dénonce le plus souvent aujourd'hui des intentions, des projets d'actes de sorcellerie et prétend ainsi s'opposer à leur aboutissement. Il n'en reste pas moins que les sorciers n'en finissent pas d'être vaincus et que les Bobo ne cessent pas de courir à leurs trousses ; a tout prendre, se consacrer aussi assidument a la contre-sorcellerie, quand on proclame avoir chasse tous les sorciers, n'est-ce pas un peu comme si l'on parlait par prétérition, comme si l'on discourait sans fin sur la sorcellerie après avoir déclaré qu'il n'y avait plus de raisons d'en parler et n'est-ce pas, en définttive, une façon dẹuisée d'entretenir une croyance?

En soi, le phénomène de la contre-sorcellerie victorieuse a des significations aussi importantes que celui de la sorcellerie agissante et $i l$ est certain que l'etude du premier jetterait bien des lumières sur le second. En l'état de nos connaissances, l'analyse des sionifications profondes du mouvement de contre-sorcellerie, tel qu'il s'est développé chez les Bobo, n'est malheureusement pas encore à notre portée ; néanmoins, puisqu'il est admis, dans le cadre de la présente publication, de faire etat de recherches qui en sont au stade exploratoire, 
nous reunirons ici les informations que nous possédons sur les divers aspects des pratiques de contre-sorcellerie.

Dans les temps anciens, la sorcellerie était très répandue et les Bobo ne disposaient pas de moyens vraiment specifiques pour lutter contre elle, ils ne connaissaient que la coutume de "l'interrogatoire du cadavre" (en bobo, sasara to dâga) - une coutume pratiquée d'ailleurs dans une grande partie de l'Afrique.

Le sasara to dâga êtait essentiellement destiné à déterminer les causes, quelies qu'elles fussent, de la mort quand cette dernière apparaissait suspecte. Par cette méthode, il pouvait être rêvélé que le mort s'êtait rendu coupable d'une faute grave et qu'il s'agissait donc d'une punition (en ce cas, on était aussi informé de l'identité de la puissance soirituelle outragée, ce qui permettait d'orqaniser les nécessaires rites expiatoires); mais, par cette méthode, on pouvait aussi découvrir que le mort était la victime d'un sorcier-empoisonneur : ce dernier était dénoncé par le cadavre lui-même et il passait "spontanément" aux aveux, avant d'être livré à la vindicte publique.

Les Bobo procédaient de la façon suivante. Le cadavre, attaché sur une civière, était hissê sur la tête de deux hormes. La scène se passait devant la maison-temple du chef de lignauge (wasa). Un prêtre ou un parent à plaisanterie (koronate) s'adressait au défunt et lui posait une série de questions précises auxquelles il était censé répondre par une impulsion irrésistible communiquée à ses porteurs et qui les poussait à faire quelques pas en avant (réponse affirmative) ou en arrière (réponse négative). On demandait par exemple au cadavre si ce qui avait causé sa mort venait de la wasa (si c'était donc une affaire concernant le lignage); si c'était une des puissances dont l'autel est dans cette méme wasa qui l'avait tue (en cas de réponse affirmative on énumérait tous les autels); on demandait si c'était les ancêtres qui avaient provoque sa mort et pour quelle raison. Si le cadavre avait toujours pousse ses porteurs en arrière, on en venait alors à la question fatidique : étaitce un homme qui était le responsable de sa mort ? si oui, chaque 
personne de la famille, puis chaque villageois passait devant le cadavre et posait la question : "Est-ce moi qui t'ai tué?" Le coupable etait designé par le mouvement voulu. Dans certaines localités, quand l'interrogatoire avait dévoilé qu'il y avait un crime, le cadavre poussait ses porteurs au long des ruelles jusqu'a la demeure de l'assassin qui etait en général tué sur place.

Cette methode de recherche des causes de la mort - qui pouvait occasionnellement permettre de découvrir des sorciers empolsonneurs - est restée longtemps employée par les Bobo et par leurs proches parents ethniques Bwa. Chez ces derniers, elle était encore attestée à l'époque ou écrivait le docteur. Cremer (entre 1910 et 1920) (1) et chez les Bobo, elle est toujours pratiquée en certains cas dans la tribu bakôma (réaion de Kuka).

Il semble néanmoins que les Bobo en soient venus à considerer que l'interrogatoire du cadavre n'était pas un moyen assez puissant pour lutter contre les sorciers. Ils s'adressèrent d'abord à certaines des puissances spirituelles majeures qu'ils réveraient et notamment à sogo, divinité de la brousse(2). Puis se répandirent les cultes d'esprits particuliers, spécialises de plus en plus dans la chasse aux sorciers.

Nous parlerons ici du culte des deux principaux esprits

(1) Cremer, J. (Dr.). Les Bobo ( a mentalité mystique).Paris, Paul Geuthner, 1927, pp. 182-188.

(2) sogo regente les forces frustes de la nature, il s'identifie à la végétation, à la brousse incultivée. Sogo s'oppose a kiri, le village, le domaine socialise, l'espace habitépar les hommes où règne ordre et paix. La brousse est pour l'homme un lieu de dangers, les forces qui y rôdent lui sont hostiles; tout a qui est négatif, inverse de l'intérêt de l'homme y prospère. La brousse est un lieu d'élection pour le sorcier qui y puise non seulement les sucs mortels des plantes sauvages, mais aussi sans doute les forces maleficues qui l'inspirent (le nycma). Pinsi, par sogo, si l'on obtient sa faveur, on peut agir sur la sorcellerie.

L'un des cultes locaux de sogo, celui du village de Ièkoro (ou Iyako) et qui porte pour cette raison le nom de $z_{y}$ ako sogo, a aoquis une grande reputation d'efficacité dans la chasse aux sorciers. Ie lyako sogo est répandu dans tout le pays bobo, mais il est aussi présent chez les Bolon et chez les Bwa (J. Cremer, op. cit. p. 190, le cite sous le nom de "Nyiulé de Lekoro"; Nyiulé est chez les Bwa l'équivalent de sogo chez les Bobo. 
chargés de detecter et de detruire les sorciers-empoisonneurs kyente : duba et vyetôgo.

I. $d u b a$.

Le culte de cet esprit est très anciennement implante en pays bobo et l'on peut dire qu'il est pratique dans la totalité des villages.

Trois varietés de duba sont connues, chacune portant le nom du village ou son culte fut inauguré et d'où il se propagea ensuite.

a) Le diriko duba (du nom d'un village disparu qui etait situé entre 'Tâguna et Duma) occupe toute la partie nord du pays bobo au-dessus d'une ligne qui passe, en les englobant, par les villages de Kuni, Worowe, Tiriko. Ie culte intéresse donc dans leur entier les tribus Tinkire, Yebè, Sâkôma, Banakôma.

b) Le mewana duba est très largement majoritaire au centre du pays dans les tribus Kurekôma et Bakôma : respectivement, régions de piriwe-Lèkoro et Bokoroniso-kuka-Mawana (son lieu de naissance). Chez les syêkôma, il est à Kurumani, Fini, Kokoroba, Zokoema et Badema. Le mawana $d u b a$ se trouve aussi en quelques points isolés de la zone du diriko duba (à Moro, trèle, Sâwê, Bagâ) et, dans la zone du zôkôma duba, à sâkoro. Le mawana duba partage enfin assez également avec le zôkôma duba tout le sud du pays bobo (tribus vorè, syakôma, bèñè et sogokirè) et 1'ensemble du pays Bolon.

c) Le zôkôma duba (du nom d'un village disparu et de localisation inconnue) a son centre principal chez les syêkôma, on le trouve notamment à Lya, Burawala, Sama, Kibe. Mais, comme nous l'avons dit, il se partage avec le mawana duba les villages bobo du sud et ceux du pays bolon.

L'autel principal de $d u b a$ est d'une forme très caractéristique et sa présence est si familière dans les villages (1) qu'on pourrait presque en faire l'emblème religieux des bobo.

(1) Sauf dans l'extrême nord du pays où l'autel de duba est à l'intérieur d'un temple. 
En fait, il y a toujours deux autels : l'un mâle, qui est le principal, et l'autre, femelle.

Le duba mâle est toujours placé a l'est du village, au voisinage des premieres maisons. Cette situation s'explique par le fait que, pour les Bobo, l'ensemble du cosmos est orienté selon un axe qui va de l'est vers l'ouest : l'est est un lieu de dangers et une source de désordres, de maléfices ; l'ouest est un havre de paix et de sécurité. Le $d u b a$ se dresse ainsi à l'est comme une sentinelle qui protégerait le village contre ses ennemis invisibles qui ont nom : maladie, sterilite, famine et... sorcellerie.

Le $d u b a$ mâle (duba sî) est une pièce monoxyle constituée par un fût rectiligne, fiche en terre à sa base et dont la partie supérieure se termine par une fourche à trois branches. Entre les branches de cette fourche est calée une poterie cylindrique munie d'un couvercle. La hauteur de la pièce de bois (qu'on appelle se le) est d'environ $1 \mathrm{~m}, 50$, le diamètre du fût 12 à $15 \mathrm{~cm}$. Les fourches et surtout le fût du duba sont ornés de sculptures. Il s'agit principalement de motifs geometriques en relief accuse, dépourvus, semble-t-il, de toute autre valeur que decorative, à l'exception toutefois d'un chanfrein rectangulaire ménage a la commissure des fourches et qui est le symbole masculin du duba. A la base du fût, on trouve les seules représentations animalières, celle du caméléon (symbole mâle) associée à celle du lézard (symbole femelle).

Le $d u b a$ femelle est appele duba $\hat{n} a$,"Epouse ( $\hat{n} a)$ de $d u b a "$. zôkôma duba est la seule varieté de duba dont l'autel femelle soit, comme les autels mâles, constitue par une sele - fût à fourche trifide. Dans ce cas, le sele femelle trouve place a l'est aux côtés de son homologue mâle; 11 ne porte naturellement que la représentation du lézard. Les deux autres variétés de duba ont leur autel femelle situé à l'ouest du villaçe, dans la zone "protégée" ; cet autel est peu visible, se presentant sous la forme soit d'un petit cône de pisé (diriko duba), soit d'un amas de cailloux (mawana duba). 
Les autels que nous venons de decrire sont les "sièges" (kware) de l'esprit duba, ce ne sont que des superstructures destinees à contenir les vrais principes actlfs. Ces "principes" sont rassembles dans la poterie supportee par les fouriches du $d u b a \quad s \hat{i}(1)$. Ils consistent en un certain nombre de tronçons de racines de l'arbre kivi (Afzelia africana) balgnant dans une eau additionnée d'un peu de bière de mil. C'est à l'intérieur de l'arbre kivi, et surtout de ses racines, que s'incorpore l'esprit duba - probablement en se diluant dans la sève.

En lui-méme, Afzelia africana est un arbre eminemment sacre pour les Bobo, comme pour de nombreuses autres populations de la zone soudanaise d'ailleurs. On notera que les racines de cet Afzelia sont réputées dangereuses, elles auraient en effet la propriete de se mouvoir spontanement, de se tordre sur ellesmêmes ou de se recourber; lorsqu'on les pose sur la tête au cours des rites, elles arracheraient les cheveux. Pour ajouter à leur virulence naturelle et aussi pour supplémenter leur pouvolr, les racines de $k i v i$ sont enduites sur toute leur longueur d'un broyat de racines de bakana humidifies a la bière de mil. Ce bakana (2) est lui-même un arbuste malfaisant dont on dit qu'il est capable de se deplacer pour barrer votre route. on attribue aux racines la propriete de provoquer chez l'homme des crises de possession et c'est là, sans nul doute, la raison pour laquelle elles sont mises en contact avec $k i v i$.

En dehors de sa présence dans la poterie de duba st, la racine de $k i v i$, soigneusement enduite de bakana, fait auss 1 office d'autel portatif et son rôle, on le verra, est de conduire et d'inspirer celui qui le porte. La racine est enroulee dans une pièce de coton blanc, étroltement serrée par des liens. A chaque extremite on attache une touffe de feuilles de nere. Plusieurs racines sont ainsi preparees, d'autres sont simplement enrobees dans une gangue d'argile. En raison de leur forme

(1) L'autel duba ña ne contient que les résidus matériels de ces principes. Ils sont déposés après leur renouvellement septennal (cf. infra) et ont, en fait, perdu toute efficacite.

(2) Plante non identifiée. 
allongee et de la touffe qui termine certaines d'entre elles, on leur donne le nom de nyâpége (de nyâga "boeuf" et de pége "queue") ou "queue de boeuf". Très nombreux sont les esprits (funyanonô) qui possedent ainsi a la fois un autel fixe et un ou des autels portatifs du type ny âpége.

Les racines de $k i v i$ (et, il faut le soulianer, celles de nombreuses autres espèces botaniques, quoique à un dearé peut-être moindre) sont donc un lieu d'élection pour la substance incorporelle des esprits. Il semble cependant que cette qualite puisse s'altérer avec le temps. Afin que les racines restent toujours en état d'abriter des forces spirituelles, il est nécessaire de procéder périodiquement à leur renouvellement. Cela donne lieu à une cérémonie qui a lieu tous les sept ans et que l'on appelle : duba suruwa dâga "coutume de la sortie (suru) de duba". A cette occasion, un nouveau sele est taillé et c'est au cours d'un rituel nocturne que les nouvelles racines de kivi sont prélevées en brousse et rapportées au villace où ont lieu sacrifices et rejouissances.

Les fonctions de $d u b a$ avaient une certaine diversité: on lui attribuait - et on lui attribue aujourd'hui encore - la naissance de nombreux enfants ; on lui reconnaissait aussi une action bienfaisante en matière d'activités agraires. Néanmoins, la fonction spécifique de $d u b a$ était la recherche et la destruction des sorciers. En ce domaine, il ne cessa d'enreaistrer des succès jusqu'au jour oũ apparut un nouvel esprit nommé vyetôgo qui devait faire preuve a'une efficacité plus qrande encore.

Les modes opératoires de $d u b a$ étant pratiquement identiques a ceux de vyetôgo, nous en traiterons à propos de ce demier. 
2. vyetägo

vyetôgo pourrait être de provenance etrangère (1); G. Chéron le disalt venir de chez les sembla (2). Les Bobo, quant a eux, s'accordent pour situer son origine à sangulema, un village limitrophe a la fois du pays sembla et du pays minyanka.

De Sanguléma, vyetôgo s'est rêpandu, au cours du $19 e$ siècle, dans la totalite des villages bobo situes au sud de la Volta noire. Vers 1900, les villageois de Sama se procurent le vyetôgo a Sanguléma. Pour la premierre fois, le culte passe outre-Volta, mais 11 faudra attendre 45 ans pour qu'il reprenne sa progression vers le nord. Silêkoro en 1945, puis Kyebant en 46 (d'ou il sera transmis a Kula, Muna et à Yakrisi en 54), Kurumani en 47, Bura en 50, Fo en 54 et sâkoro en 56 se rendent acquéreurs du vyetôgo à Sama. Entre temps, en 1952, Fini est allé chercher le culte à Sanguléma même pour le transmettre à Mana en 56 et Kuka en 57. De son côté, Wofura rapporte en 55 le vyetôgo de Sanguléma et le donne à Tuma en 57. En 1959 enfin, GI, Be et Felewe, les villages les plus septentrionaux étaient en train d'adopter vyetôgo. Depuis, le culte a achevé de se généraliser.

L'autel de vyetögo est constitue toujours par deux poteaux a triple fourche supportant des poteries closes ; l'un est male, l'autre est femelle et lis sont placés côte a côte. Ces sele ressemblent beaucoup au duba sî, néanmoins ils s'en distinguent par le fait qu'ils ne sont pratiquement pas sculptés et qu'ils se dressent toujours à l'interieur du village. Les principes actifs de duba se retrouvent chez le vyetôgo et chez ce dernier, ils existent egalement sous la forme de nyâpége.

(1) En ce cas, duba, qui lui est anterrieur chez les Bobo, mais qui lui ressemble tant, aurait eté lui aussi importe. Pour notre part, une commune origine minyanka nous paraft une hypothèse vraisemblable. Les faits de possession qui caractérisent le culte tant de duba que de vyetôgo sont très typiques de la religion minyanka, en revanche, ils sont absents dans le culte des figures centrales de la religion bobo (Dwo en particulier).

(2) Chêron, G. "Les Bobo-fing". Annuaire et Mémoires de Com. d'ét. hist. et sc. de Z'A.O.F., 1916, p. 257. 
Enfin, le renouvellement, ici auss1, est septennal.

Ce qui fait pour nous l'intérêt de vyetôgo, ce sont, bien entendu, les modalités de son action contre les sorciers.

Lorsque se produit le décès d'un individu jeune et qui etait apparemment de bonne constitution, 11 convient de s'informer sur les causes de sa mort. A plus forte raison faut-il le faire si l'on avait observe chez lui une alteration suspecte des facultês. Une requête est présentée d'abord aux ancêtres (bore). S'ils restent muets (les poulets du sacrifice mourant de telle façon qu'on ne puisse en inferrer une réponse précise), on est en droit de redouter le pire. Il faut alors s'adresser a sogo (sogo to, "interroger sogo") car il regente, nous l'avans dit, le monde de la brousse et ses forces obscures; personne n'est plus apte a reconnaitre et à dénoncer l'oeuvre de sorcellerie. sogo révèle donc les actes criminels, mais en aucun cas il ne désigne les coupables et moins encore 11 ne les punit lui-même : c'est là la tâche exclusive de vyetôgo. sogo établit le diagnostic, vyetôgo "opère".

Informes du crime perpetré, les parents du mort vont devant l'autel de vyetôgo. Ils implorent sa vengeance et offrent une poule en sacrifice. Ouelque temps s'êcoule avant que, d'une façon tout a fait soudaine et imprêvue, une personne entre brutalement en transes. La possession ne peut se produire qu'hors du village, en pleine brousse, et la crise est susceptible, aff1rme-t-on, de toucher $n$ 'importe qui. Dès les premiers symptômes, l'homme possédé court au village et s'empare d'un nyâpége de vyetogo, il s'arme aussi d'une lance. Titubant, il se rend chez le chef de famille du defunt et un dialogue s'établit. Le chef de famille explique l'affaire et demande a l'homme en qui s'incarne désormais vyetôgo de trouver le coupable. Le possédé acquiesce en frappant le sol de son nyâpége et en sortant de sa bouche écumeuse des sons qui sont souvent comparés aux hennissements du cheval (on dit, ici comme ailleurs, que le possede est le "cheval" de l'esprit). Les choses sont alors très rapides: le possédé dévale en courant les ruelles et $s^{\prime}$ arrête brusquement devant une porte ; il entre et se dirice droit sur la cachette ou l'empoisonneur a placé son matériel, fioles de poison, mortiers, 


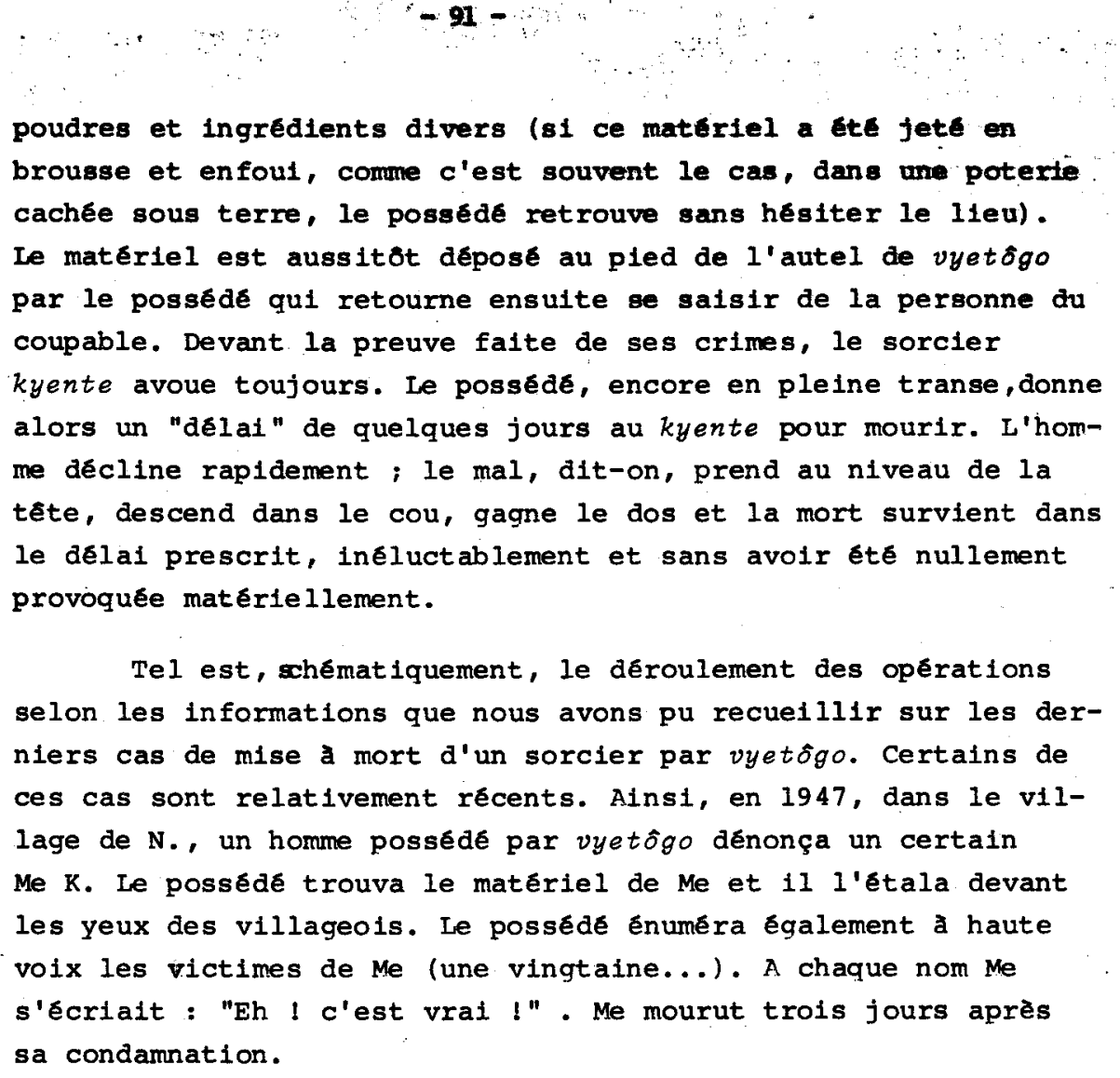

Ayant rassemble ces quelques falts, au moins pouvons nous mesurer mieux la complexité de phénomènes qui concernent non seulement le domaine de la sorcellerie, mais aussi celui de la possesston et d'une certaine façon, celui de la divination. 
$-92-$

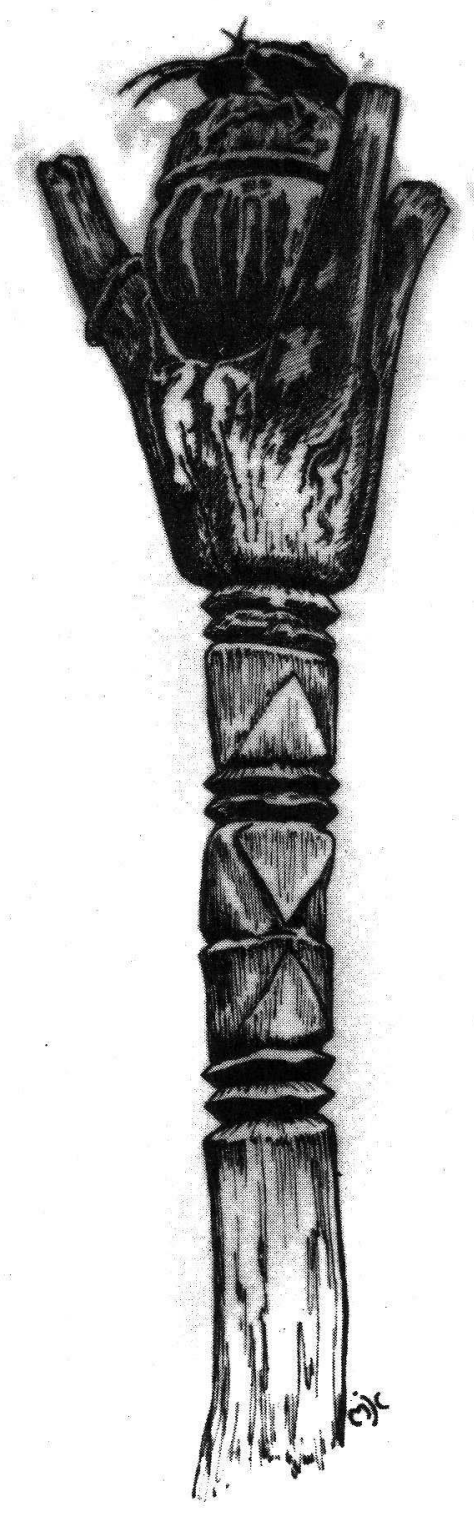

Vyetôgo 


\section{DISCUSSIOR}

M. CARTRY - Quelle est la nature des polsons préparés. Sont-ils vraiment très toxiques?

G. LE MOAL - Il s'agit de poisons vegetaux très actifs. On y joint souvent des fragments de gros intestin d'hyène, or 11 est scientifiquement tabli que cela est très toxique: Je ne parle pas des ingredients divers, tels que les restes humains en decomposition, qui ne doivent pas être très digestes !

G. DIETERLEN- Au sujet de l'orientation de l'espace : beaucoup de choses viennent de l'est. Chante-t-on : "le masque est venu de l'est.."?

G. $L E$ MOAL - Oui, chez les Bobo, les masques sont fabriqués en brousse a $l$ 'est du village. Ils operrent en traversant le village d'est en ouest. Tout est orienté est-ouest, de ce fait l'est est la source de toutes choses. Il faut regarder vers l'est, 11 faut aussi contrôler et canaliser, en quelque sorte, les forces qui viennent de l'est - c'est pourquol duba se dresse a l'est du village.

L. de HEUSCH - Pour quelle raison 1'Afzelia a-t-il un rôle aussi important?

G. $L E$ MOAL - L'Afzelia est le vecteur de forces spirituelles d'importance majeure. Les autels bobo, pratiquement tous, sont fondes sur des composants vegetaux, or il y a un rapport étroit entre la taxonomie bobo des plantes et la classification, la hierarchie, des entites spirituelles. Il $y$ a tout un jeu de correspondances classificatoires entre les qualités du réceptacle et la nature de la force qui s'y incorpore.

M. CARTRY - Est-ce que tous les Afzelia sont sacrés ? Chez les Gourmantche, ce $n$ 'est le cas que pour certains d'entre eux.

G. $L E M O A L$ - Oui, c'est l'espèce tout ent1ère qui est sacrée. On ne coupe un $A f z e l i a$ que pour des raisons religieuses, pour y tailler un masque par exemple (voir mon film "Le grand masque Molo").

N. ECHARD - Est-ce que la connaissance de la preparation des poisons est le fait de certains groupes soclaux particuliers ?

G. LE MOAL - Non, c'est une connaissance dont les principes de base sont accessibles a tous. Le sorcier $n$ 'est pas nécessairement plus 
savant que quiconoue en matière de poisons, mais, luf, il en falt un usage systematiquement criminel. D'allieurs, on est sorcier par la philosophie de son acte et non par un plus ou moins grand savoir.

J.F. VINCENT - II serait intéressant de voir comment l'action du vyetógo a remplace l'action du duba.

G. $L E M O A L$ - En effet, il y a la une substitution que je ne sals pas expliquer. duba etait et reste encore, semble-t-11, en mesure de detecter les sorciers, mais sa vocation est plus etendue, plus pacifique, plus neutre que celle de vyetôgo qui s'attaque seulement à ce qui trouble l'ordre social.

J.F.VINCENT - L'action de vyetôgo ressemble un peu a celle des $n k i s i$.

L. DE HEUSCH - Certains nkisi ont un rolle de protection contre la sorcellerie. Mais, 1ls ont un rôle individuel et non collectif. Or ici, ils ont une fonction collective. Il y a aussi un lien avec la possession, car il arrive que la puissance qui est dans le $n k i$ i $i$ puisse provoquer la possession, mais là encore dans un cadre individuel plutót que collectif.

M. CARTRY - Il semble que les vyetôgo aient des "proprietaires" et qu'on puisse les acquérir. Est-ce que le proprietaire accepte de mettre son autel a la disposition de la victime?

G. $L E M O A L$ - Il y a deux plans : le communautaire (foroba), c'est-à-dire ce qui est partage par tous les membres de la communaute villageolse, et le lignager ( $z a k a n e$ ). vyetôgo. appartient au liq̣nage parmi lequel s'est trouve celui qui, soit en a eu la révelation, soit a procéde a son acquisition. vyetógo est donc zakane, "privé" en quelque sorte. Mais, dans ses activités, vyetôgo opère au niveau de la communauté (foroba) et au bénefice de tous. 